\title{
Expiratory model-based method to monitor ARDS disease state
}

\author{
Erwin J van Drunen', Yeong Shiong Chiew¹, J Geoffrey Chase', Geoffrey M Shaw², Bernard Lambermont ${ }^{3}$,
} Nathalie Janssen ${ }^{3}$, Nor Salwa Damanhuri ${ }^{1}$ and Thomas Desaive ${ }^{4^{*}}$

\footnotetext{
* Correspondence: tdesaive@ulg.ac.be ${ }^{4}$ University of Liège, Liège, Belgium Full list of author information is available at the end of the article
}

\begin{abstract}
Introduction: Model-based methods can be used to characterise patient-specific condition and response to mechanical ventilation (MV) during treatment for acute respiratory distress syndrome (ARDS). Conventional metrics of respiratory mechanics are based on inspiration only, neglecting data from the expiration cycle. However, it is hypothesised that expiratory data can be used to determine an alternative metric, offering another means to track patient condition and guide positive end expiratory pressure (PEEP) selection.
\end{abstract}

Methods: Three fully sedated, oleic acid induced ARDS piglets underwent three experimental phases. Phase 1 was a healthy state recruitment manoeuvre. Phase 2 was a progression from a healthy state to an oleic acid induced ARDS state. Phase 3 was an ARDS state recruitment manoeuvre. The expiratory time-constant model parameter was determined for every breathing cycle for each subject. Trends were compared to estimates of lung elastance determined by means of an end-inspiratory pause method and an integral-based method. All experimental procedures, protocols and the use of data in this study were reviewed and approved by the Ethics Committee of the University of Liege Medical Faculty.

Results: The overall median absolute percentage fitting error for the expiratory time-constant model across all three phases was less than $10 \%$ for each subject, indicating the capability of the model to capture the mechanics of breathing during expiration. Provided the respiratory resistance was constant, the model was able to adequately identify trends and fundamental changes in respiratory mechanics.

Conclusion: Overall, this is a proof of concept study that shows the potential of continuous monitoring of respiratory mechanics in clinical practice. Respiratory system mechanics vary with disease state development and in response to MV settings. Therefore, titrating PEEP to minimal elastance theoretically results in optimal PEEP selection. Trends matched clinical expectation demonstrating robustness and potential for guiding MV therapy. However, further research is required to confirm the use of such real-time methods in actual ARDS patients, both sedated and spontaneously breathing.

Keywords: Mechanical ventilation, Model-based methods, Expiration, ARDS, Intensive care, Time constant

\section{Biomed Central}

(c) 2013 van Drunen et al.; licensee BioMed Central Ltd. This is an Open Access article distributed under the terms of the Creative Commons Attribution License (http://creativecommons.org/licenses/by/2.0), which permits unrestricted use, distribution, and reproduction in any medium, provided the original work is properly cited. 


\section{Background}

Patients suffering from severe respiratory insufficiency, such as acute respiratory distress syndrome (ARDS) [1] are admitted to the intensive care unit (ICU) and require mechanical ventilation (MV) for breathing support. ARDS is associated with a loss of functional lung units resulting in a stiffer lung [2]. The severity of ARDS is typically measured as the ratio of the arterial partial pressure of oxygen divided by the fraction of inspired oxygen $\left(\mathrm{PaO}_{2} / \mathrm{FiO}_{2}\right)$ [1]. Clinicians offer a supportive environment to ARDS patients by applying positive end expiratory pressure (PEEP) to aid recovery by improving gas exchange and maintaining recruitment during subsequent breathing cycles [3-6]. Given the impact of MV on cost and length of stay [3], ensuring optimal PEEP would have significant impact.

Modelling the respiratory mechanics of MV patients can potentially provide a noninvasive method to obtain clinically and physiologically useful information to guide MV therapy [7-10]. Conventional metrics of respiratory mechanics are estimated based on the mechanics of breathing during inspiration, often neglecting expiratory data. However, passive expiration can be used to determine a metric based on the expiratory flow profile [11], potentially offering insight into respiratory mechanics in situations where conventional inspiratory metrics will not work. Real-time monitoring of model-based respiratory mechanics throughout treatment for ARDS will provide unique descriptions of the patient's disease progression and response to MV [12-15], offering the ability to guide patient-specific MV.

\section{Method}

\section{Time-constant model}

The single compartment lung model is defined [16]:

$$
P_{a w}(t)=R_{r s} \times Q(t)+E_{r s} \times V(t)+P_{0}
$$

where $P_{a w}$ is the airway pressure, $t$ is time, $R_{r s}$ is the series resistance of the endotracheal tube and the conducting airway, $Q$ is the air flow, $E_{r s}$ is the respiratory system elastance, $V$ is the lung volume and $P_{O}$ is the offset pressure.

Inspiration and expiration are different physiological processes. Expiration is essentially the passive unloading of the inspired tidal volume over a resistance at a constant ventilator applied pressure $\left(P_{a w}=\mathrm{PEEP}\right)$ with $P_{0} \approx$ PEEP $[17,18]$. Noting that volume is the integral of flow with respect to time, Eq. 1 in expiration becomes:

$$
P E E P=R_{r s} \times Q(t)+E_{r s} \times \int_{0}^{t} Q\left(t^{\prime}\right) d t^{\prime}+P E E P
$$

Differentiating Eq. 2 yields:

$$
0=R_{r s} \times \frac{d Q(t)}{d t}+E_{r s} \times Q(t)
$$

Dividing Eq. 3 by the resistance, $R_{r s}$, yields a simple ordinary differential equation:

$$
\frac{d Q(t)}{d t}+\frac{E_{r s}}{R_{r s}} \times Q(t)=0
$$

Solving Eq. 4 yields:

$$
Q(t)=Q_{o} e^{-t / \tau}=Q_{o} e^{-K t}
$$


where $Q_{o}$ is the value of maximum expiratory flow and $\tau=1 / K=R_{r s} / E_{r s}$ is the system time-constant [11].

If the resistance, $R_{r s}$, is assumed constant [19], then $K$ is directly proportional to $E_{r s}$ where an increasing parameter $K$ implies a less compliant lung as ARDS progresses, as shown in Figure 1.

\section{Experimental data}

This study examines three fully sedated experimental ARDS piglets ventilated using Engström CareStation ventilators (Datex, General Electric Healthcare, Finland) with a volume controlled, square flow profile and a constant $\mathrm{FiO}_{2}$ of 0.5 [20]. An Eview data acquisition device with a constant sampling rate was used to record the airway pressure and flow data continuously throughout the trials.

The subjects underwent three experimental phases. Phase 1 was a staircase recruitment manoeuvre with PEEP settings at $5-10-15-20-15-10-5 \mathrm{cmH}_{2} \mathrm{O}$ while the subject was in a healthy state. Phase 2 was a progression from a healthy sedated state to an oleic acid induced ARDS state at a constant PEEP of $5 \mathrm{cmH}_{2} \mathrm{O}$. Phase 3 was a staircase recruitment manoeuvre with PEEP settings at $5-10-15-20-15-10-$ $5 \mathrm{cmH}_{2} \mathrm{O}$ after the subject was diagnosed with ARDS. Thus, an initial recruitment manoeuvre provides a comparison to a healthy state. Each subject has between 1600 and 3500 recorded breathing cycles across all three phases for a total of 6800 breathing cycles. All experimental procedures, protocols and the use of data in this study were reviewed and approved by the Ethics Committee of the University of Liege Medical Faculty.

\section{Model fitting}

The values of $K$ and $Q_{o}$ are determined from the least-squares best fit of the timeconstant model (Eq. 5) to the expiratory flow data. Determining $Q_{o}$ simultaneously with $K$ leads to robust parameter identification as the effect of outliers at the beginning of expiration is reduced. During expiration, the density of data points increases as expiration progresses because the rate of change of flow decreases. Therefore, the model fit becomes more constrained towards the end of expiration. When combined

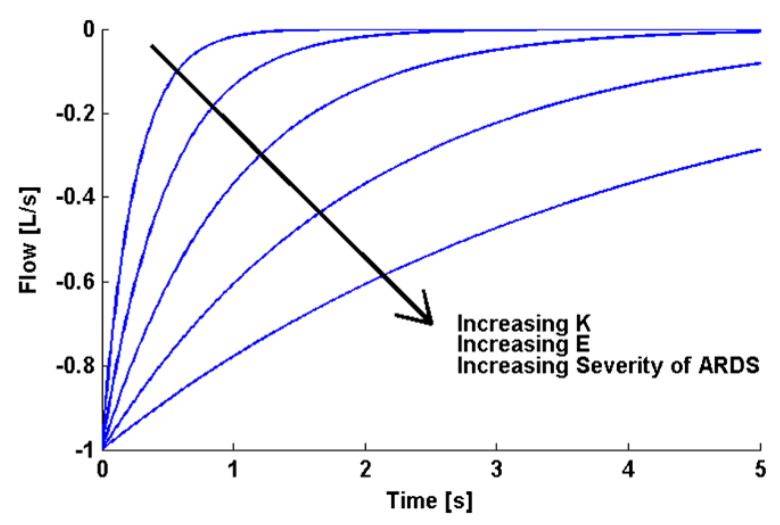

Figure 1 Example of how changes in expiratory flow profile over time may be used to determine changes in a patients' disease state, assuming $R_{r s}$ is constant. 
with the presence of a long portion of near-constant flow at the end of expiration caused by resistance in the ventilator's expiratory valve [11], poor model fitting can occur. Hence, model fitting is limited to the time span required for the respiratory system to reach $95 \%$ of its equilibrium value, as illustrated in Figure 2 (Top).

The expiratory pressure data recorded in this study shows a sudden decrease to just above PEEP, followed by a small trailing portion as shown for a representative breath in Figure 2 (Bottom). This data is expected to contain no physiologically useful information since it is measured downstream of the ventilator's expiratory valve. Furthermore, the change in pressure from 1.12 seconds to the end of expiration is minor. This outcome further justifies the approach of only considering flow data for model fitting during expiration.

\section{Validation}

The time-constant model parameter, $K$, is determined continuously for every breathing cycle for each subject at different experimental phases. The model is validated by comparing trends in $K$ to trends obtained using an end-inspiratory pause (EIP) method and trends obtained using an integral-based method. Both methods determine unique inspiratory values of respiratory elastance and resistance for each breathing cycle,

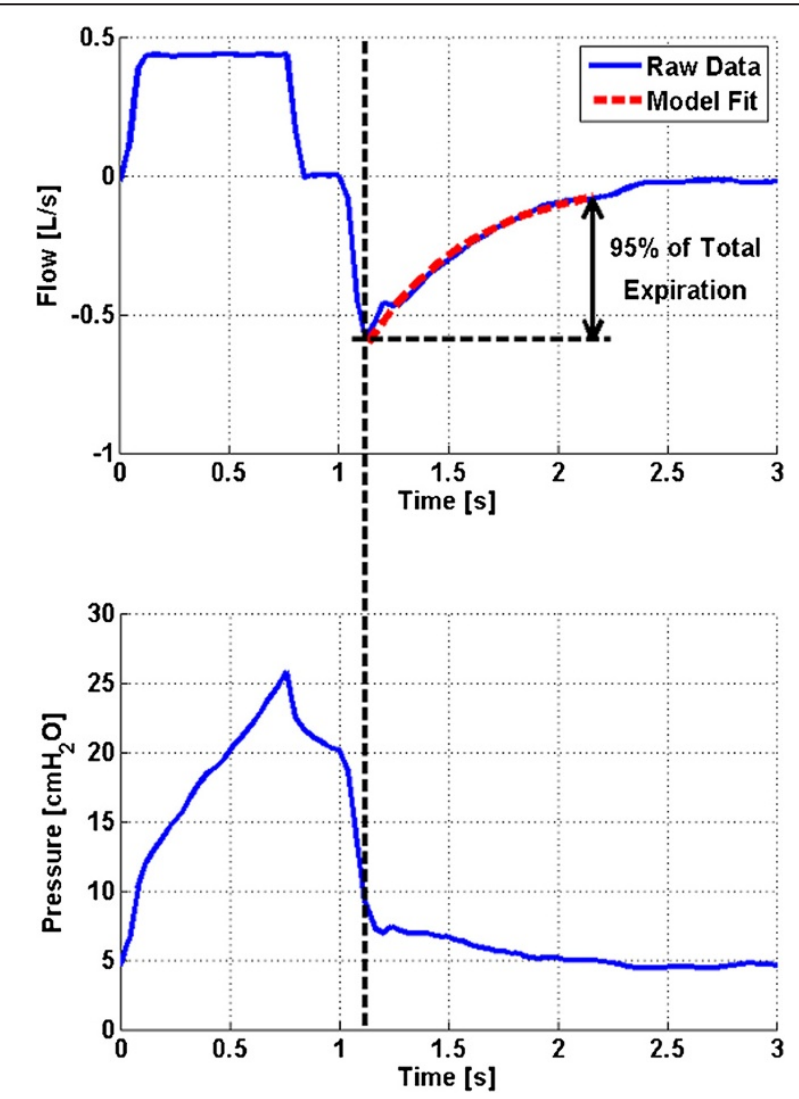

Figure 2 Top: Flow profile of a single breathing cycle with exponential model fit to expiratory flow data. Bottom: Pressure profile of a single breathing cycle at PEEP $=5 \mathrm{cmH}_{2} \mathrm{O}$. In this case, pressure data after 1.12 seconds is physiologically meaningless. 
providing more insight into respiratory mechanics than the single lumped parameter, K. In particular:

1 The EIP method determines metrics of respiratory mechanics directly from the Engström CareStation ventilator (Datex, General Electric Healthcare, Finland) which automates a short EIP during controlled MV [21-23]. The zero-flow phase during EIP omits the resistance component in Eq. 1 and prolongs inspiration, allowing the inspired tidal volume $\left(V_{t}\right)$ to distribute evenly in the lung. The resulting pressure after the EIP is called the plateau pressure, $P_{\text {plat }}$, and can be used to estimate static ventilation elastance, $E_{\text {static }}$, as shown in Eq. 6 . Equally, the pressure difference between peak inspiratory pressure (PIP) and $P_{\text {plat }}$ can be used to calculate static airway resistance, $R_{\text {static }}$, as shown in Eq. 7 .

$E_{\text {static }}=\left(P_{\text {plat }}-\mathrm{PEEP}\right) / V_{t}$

$R_{\text {static }}=\left(\mathrm{PIP}-P_{\text {plat }}\right) / Q$

2 Eq. 8 describes an integral-based method [24] to estimate breath-specific values of the respiratory elastance, $E_{r s}$, and respiratory resistance, $R_{r s}$. The recorded inspiratory pressure and flow data for each breath is used to determine values that best fit the single compartment lung model defined in Eq. 1. Integral-based parameter identification is similar to multiple linear regression where using integrals significantly increases robustness to noise $[19,24]$.

$$
\int P_{a w}(t)=R_{r S I B} \times \int Q(t)+E_{r s I B} \times \int V(t)+\int P_{0}
$$

Thus, $K$ can be compared with, $E_{\text {static }}$ and $R_{\text {static }}$, as well as, $E_{r s I B}$ and $R_{r s I B}$, both of which aim to capture the true elastance $(E)$ and resistance $(R)$ of the lung. This validation process is summarised graphically in Figure 3.

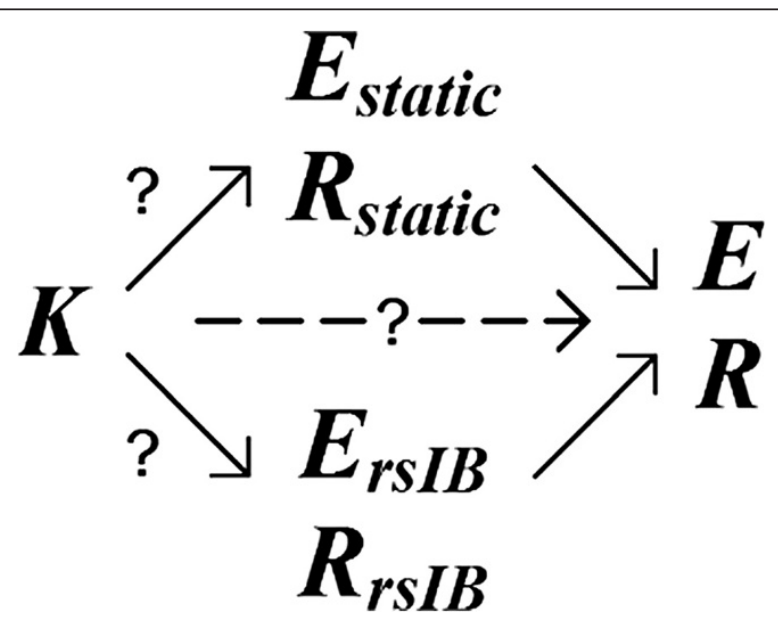

Figure 3 Graphic representation of the relationship between various metrics of respiratory mechanics. 


\section{Results and discussion}

\section{Fitting error}

For each breath, the time-constant model and both validation methods are compared to recorded flow and pressure data respectively. In particular, the time-constant model parameters, $K$ and $Q_{o}$, are substituted into Eq. 5 and the calculated flow is compared to the measured expiratory flow data used during model fitting. In the case of both validation methods, the estimated parameters, $E_{\text {static }}$ and $R_{\text {static }}$, and, $E_{r s I B}$ and $R_{r s I B}$, are substituted into Eq. 1 and the calculated pressure is compared to the measured inspiratory pressure data. Median and inter-quartile range (IQR) absolute percentage fitting errors are reported in Table 1. The integral-based method produced the lowest overall median fitting error for each subject (1.97\%, $1.55 \%$ and $2.26 \%$ for Subjects 1, 2 and 3 respectively). The timeconstant model produced the second lowest overall median fitting error for Subjects 2 and 3. All overall median fitting errors were within likely measurement errors of 3-10 \%.

\section{Healthy State Recruitment Manoeuvre - Phase 1}

During the recruitment manoeuvre, $K$ closely follows the trends of both $E_{r s I B}$ and $E_{\text {static }}$ for all three subjects, as shown in Figure 4. Estimates of resistance remain relatively constant across the recruitment manoeuvre justifying the use of the lumped parameter $K$ during this phase.

\section{Disease Progression - Phase 2}

Inter-subject differences in ARDS progression over time can be seen in Figure 5 and indicate a variable response to oleic acid to induce ARDS, as well as a variable response to MV for each subject [25-28]. After oleic acid injection, it was found that $E_{r s I B}, E_{\text {static }}$ and $K$ in Subjects 1 and 2 all followed similar trends. Each parameter shows a slow

Table 1 Model fitting errors for each $\mathrm{model} / \mathrm{method}$

\begin{tabular}{|c|c|c|c|c|c|}
\hline & \multirow[b]{2}{*}{ Subject } & \multicolumn{4}{|c|}{ Absolute percentage fitting error (Median, [IQR]) [\%] } \\
\hline & & Phase 1 & Phase 2 & Phase 3 & Overall \\
\hline \multirow[t]{6}{*}{ Time-Constant Model } & 1 & 5.01 & 7.24 & 5.19 & 6.99 \\
\hline & & {$[2.24-9.89]$} & {$[3.74-12.58]$} & {$[2.50-8.96]$} & {$[3.55-12.21]$} \\
\hline & 2 & 3.69 & 3.66 & 5.84 & 3.83 \\
\hline & & {$[1.68-7.58]$} & {$[1.59-6.78]$} & {$[2.77-9.62]$} & {$[1.67-7.12]$} \\
\hline & 3 & 4.38 & 4.37 & 10.57 & 4.72 \\
\hline & & {$[1.90-8.71]$} & {$[2.00-8.41]$} & {$[5.11-17.29]$} & [2.13-9.34] \\
\hline \multirow[t]{6}{*}{ EIP Method } & 1 & 2.61 & 3.85 & 4.07 & 3.77 \\
\hline & & {$[1.25-4.87]$} & [1.85-6.99] & {$[1.75-7.83]$} & [1.80-6.92] \\
\hline & 2 & 5.83 & 5.31 & 6.81 & 5.48 \\
\hline & & {$[3.28-10.26]$} & [2.69-9.13] & [3.84-9.91] & [2.83-9.34] \\
\hline & 3 & 5.59 & 6.32 & 12.71 & 6.61 \\
\hline & & [3.58-9.91] & {$[3.51-11.04]$} & {$[6.51-24.32]$} & [3.65-11.69] \\
\hline \multirow[t]{6}{*}{ Integral-Based Method } & 1 & 1.42 & 1.98 & 2.31 & 1.97 \\
\hline & & {$[0.59-3.18]$} & [0.92-3.84] & {$[1.03-4.12]$} & [0.90-3.81] \\
\hline & 2 & 1.81 & 1.48 & 2.04 & 1.55 \\
\hline & & {$[0.85-3.27]$} & {$[0.71-2.86]$} & {$[0.93-3.43]$} & [0.73-2.97] \\
\hline & 3 & 1.31 & 2.23 & 4.99 & 2.26 \\
\hline & & {$[0.68-2.36]$} & {$[0.95-4.95]$} & {$[2.14-8.59]$} & {$[0.96-5.11]$} \\
\hline
\end{tabular}



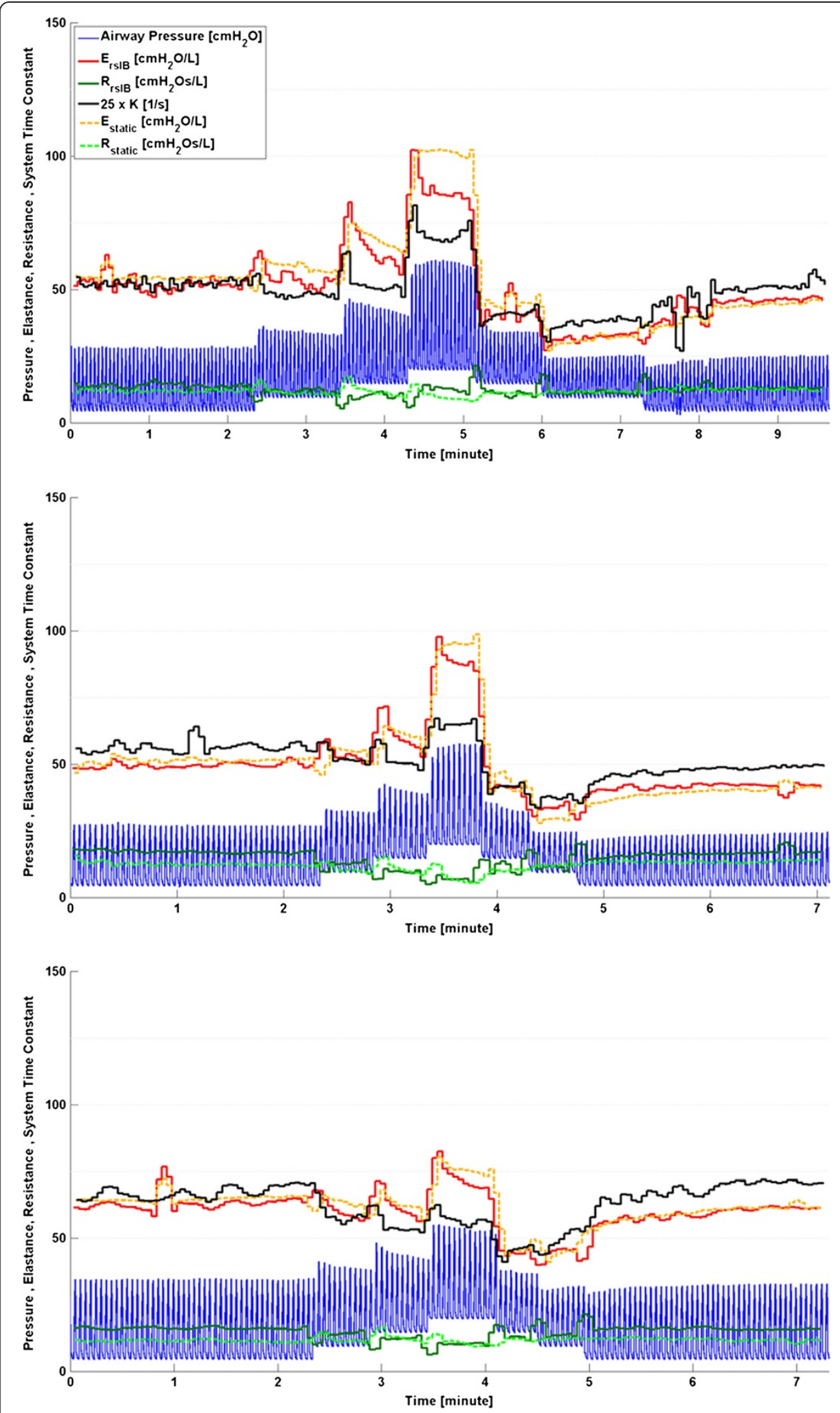

Figure 4 Respiratory system mechanics monitoring during phase 1, healthy state recruitment manoeuvre. Note that values of $K$ have been scaled for clarity and serve only as an indication for trend comparison. Subject 1 (Top), Subject 2 (Middle) and Subject 3 (Bottom). 


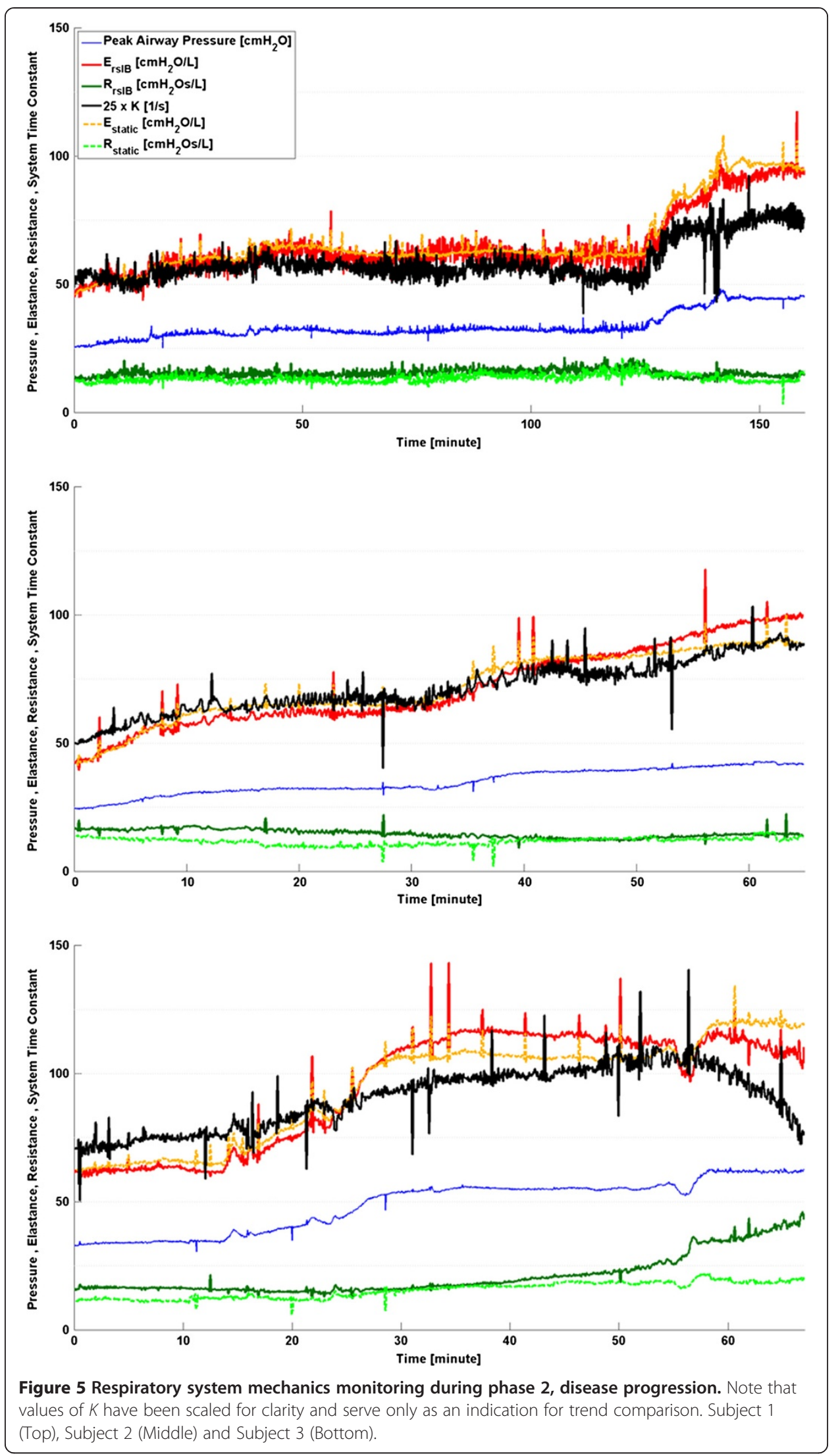


change, followed by a rapid change as ARDS develops, where increasing elastance implies the lung is becoming stiffer [2,29]. However, in Subject 3, the trend of $K$ does not follow either $E_{r s I B}, E_{\text {static }}$. This lack of correlation may be a subject-specific response due to the increasing severity of ARDS closing some of the respiratory system airways, thereby increasing the resistance [2]. Since $K=E_{r s} / R_{r s}$, an increasing resistance would result in a decreasing $K$, consistent with that shown in Figure 5 . In addition, because the resistance is varying for Subject 3, no information about the lung elasticity can be directly gained from $K$ during this phase. This result highlights the importance of the assumption of constant resistance for accurate tracking of disease progression when using this method.

\section{ARDS state recruitment manoeuvre - Phase 3}

Subject responses during phase 3 vary significantly as shown in Figure 6. Subject 1 has the highest variation in $E_{r s I B}, E_{\text {static }}$ and $K$, while Subject 3 does not show any significant change in either $E_{r s I B}$ or $K$. Trends of $E_{r S I B}, E_{\text {static }}$ and $K$ for both Subjects 1 and 2 agree, and show a definite response to the recruitment manoeuvre. Subject 3 , and to a lesser extent Subject 2, show a decrease in $R_{r s I B}$ during increasing PEEP titration and an increase in $R_{r S I B}$ during decreasing PEEP titration, suggesting that increasing PEEP opens the respiratory system airways $[12,14,30]$. This change of airway resistance indirectly affects the estimated parameters determined by the integral-based method. Furthermore, Subject 3 has the highest PIP across all three phases. This is because a higher inspiratory pressure is required to counter the effect of a larger body mass (see Table 2). However, Subject 3 also has the highest severity of ARDS as shown in Table 2. Thus, it is possible that factors aside from a variable respiratory resistance may influence the response. This result highlights both the significant inter-subject variability, and the need for subject-specificity in a model-based approach.

During PEEP titration in phase 3, respiratory elastance drops to an overall minimum at a specific PEEP for each subject (PEEP $=15 \mathrm{cmH}_{2} \mathrm{O}$ for Subject 1, PEEP $=10-15$ $\mathrm{cmH}_{2} \mathrm{O}$ for Subject 2 and PEEP $=15-20 \mathrm{cmH}_{2} \mathrm{O}$ for Subject 3). Because recruitment is a function of PEEP and time [31,32], true minimal $E_{r s I B}, E_{\text {static }}$ and $K$ can only be determined after a stabilisation period at each PEEP level. Decrease of elastance over time to a specific minimum can be described by increasing recruitment and/or the lung's viscoelastic properties, which causes hysteresis [33,34]. Setting PEEP at minimum elastance theoretically benefits ventilation by maximising recruitment, reducing work of breathing and avoiding overdistension $[12,14,15,35]$. It was also found that decreasing PEEP titration resulted in lower overall $E_{r S I B}, E_{\text {static }}$ and $K$ compared to increasing PEEP titration, as shown in Figure 6. When PEEP is increased to a higher level, recruitment, as well as potential lung overstretching, occurs. However, after PEEP is reduced, the lung remains more compliant, as expected clinically after such a recruitment manoeuvre.

\section{Trend comparison}

Performance was assessed by trend correlation coefficient $\left(R^{2}\right)$ across all three phases where comparison between $K$ and $E_{\text {static }}$, and $K$ and $E_{r S I B}$ values were made for each breathing cycle. From Figure 7, it can be seen that Subject 3 has the lowest correlation coefficients across both validation metrics, while Subject 2 has the highest correlation coefficients as expected from the observation in Figures 4, 5 and 6. In this study, Subject 3 

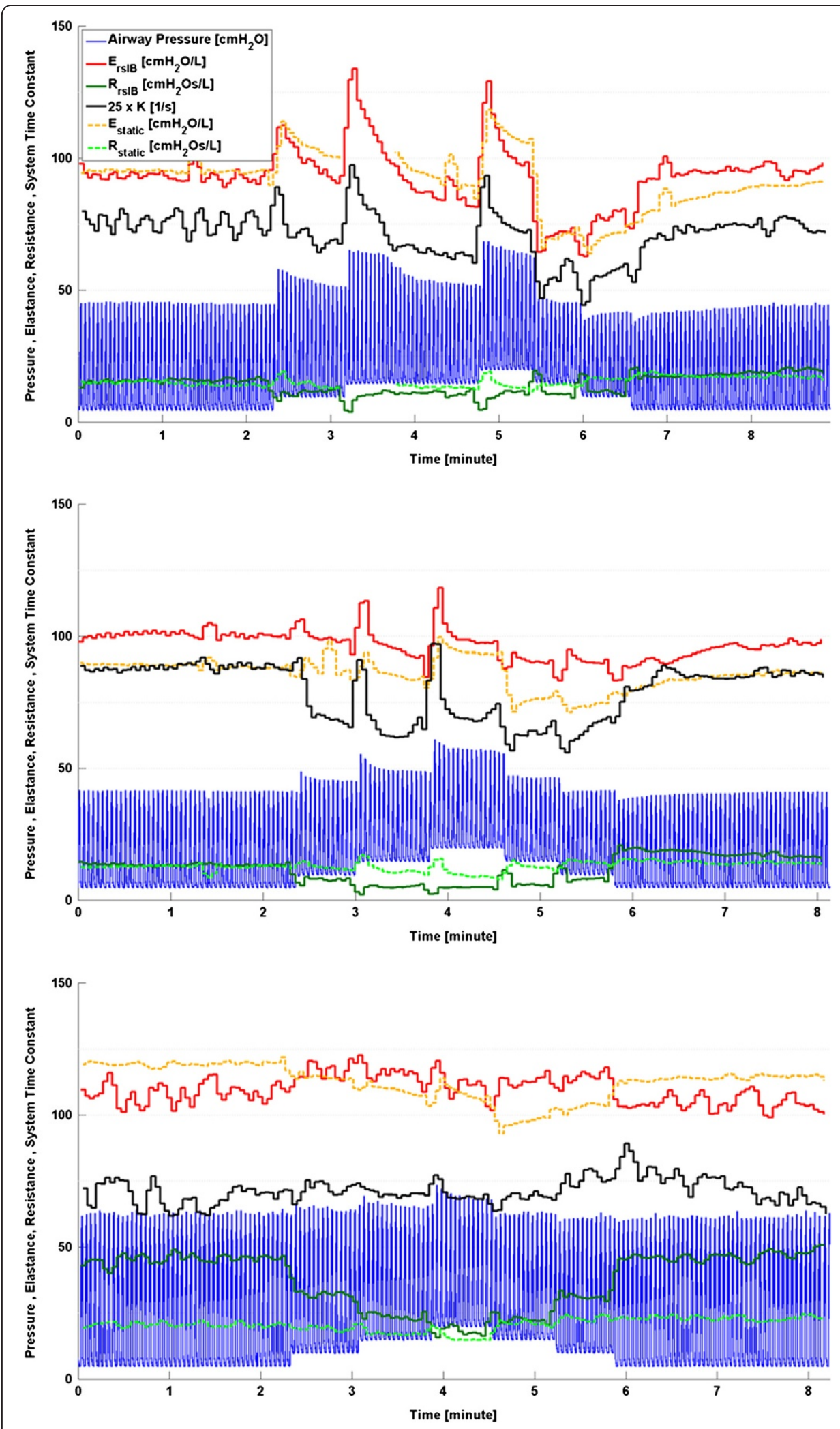

Figure 6 Respiratory system mechanics monitoring during phase 3, disease state recruitment manoeuvre. Note that values of $K$ have been scaled for clarity and serve only as an indication for trend comparison. Subject 1 (Top), Subject 2 (Middle) and Subject 3 (Bottom). 
Table 2 Body mass and $\mathrm{PaO}_{2} / \mathrm{FiO}_{2}$ ratio (during ARDS state recruitment manoeuvre) for each subject

\begin{tabular}{ccc}
\hline Subject & Mass $[\mathbf{k g}]$ & $\mathrm{PaO}_{\mathbf{2}} / \mathbf{F i O} \mathbf{2}_{\mathbf{2}}$ (Phase 3) $[\mathrm{mmHg}]$ \\
\hline $\mathbf{1}$ & 24.0 & 126.6 \\
\hline $\mathbf{2}$ & 20.3 & 183.6 \\
\hline $\mathbf{3}$ & 29.6 & 113.6 \\
\hline
\end{tabular}

has the largest body mass and reached the highest severity of ARDS while Subject 2 has the lowest body mass and reached lowest severity of ARDS. Thus, it is possible that increased body mass and/or severity of ARDS may influence the physiological process of expiration, leading to a lower observed correlation between the system time-constant, $K$, and the elastance of the lung. However, due to the limited number of trials in this study, this conclusion is limited in its impact and warrants further investigation.

\section{Outcomes}

The time-constant model has demonstrated its performance in continuous monitoring of respiratory mechanics as disease state progresses in fully sedated experimental piglets. The overall median fitting error was comparable to that obtained using the EIP method. However, variable respiratory resistance may lead to a lower correlation between the system time-constant, $K$, and the elastance of the lung. The resistance was found to vary in Subject 3 during phase 2 and Subjects 2 and 3 during phase 3, based on the results of the integral-based method. Thus, a larger study cohort is required to further validate this method. However, it should be noted that if resistance is varying, it can be identified and accounted for. In general, the time-constant model was able to provide clinically relevant physiological insight not readily available at the bedside to guide MV therapy.

One potential application of the time-constant model is to estimate respiratory mechanics of spontaneously breathing patients which have individual breathing efforts aside from ventilator support [36], significantly altering the respiratory mechanics. In this case, oesophageal pressure measurements are required to determine patient-specific respiratory mechanics during inspiration [16]. However, this technique is considered uncomfortable for the patient and its application is limited in daily monitoring despite its potential to guide MV for ARDS patients [37,38]. Expiration is hypothesised to be primarily or completely passive, regardless of whether the patient is sedated or spontaneously breathing. Thus, muscle activity is assumed to be absent or relatively minimal [11,36]. Therefore, it may be possible to determine real-time lung parameters for spontaneously breathing patients without additional measuring tools, opening up the clinical applicability of a model-based approach to guiding MV therapy. However, application of the time constant model in tracking respiratory mechanics in spontaneously breathing patients warrants further investigation.

\section{Limitations}

Pathogenesis of ARDS animal models are more consistent where the methods of developing ARDS is known and controlled. In contrast, ICU patients are more variable as the causes of disease are different with greater inter-patient variability in response 

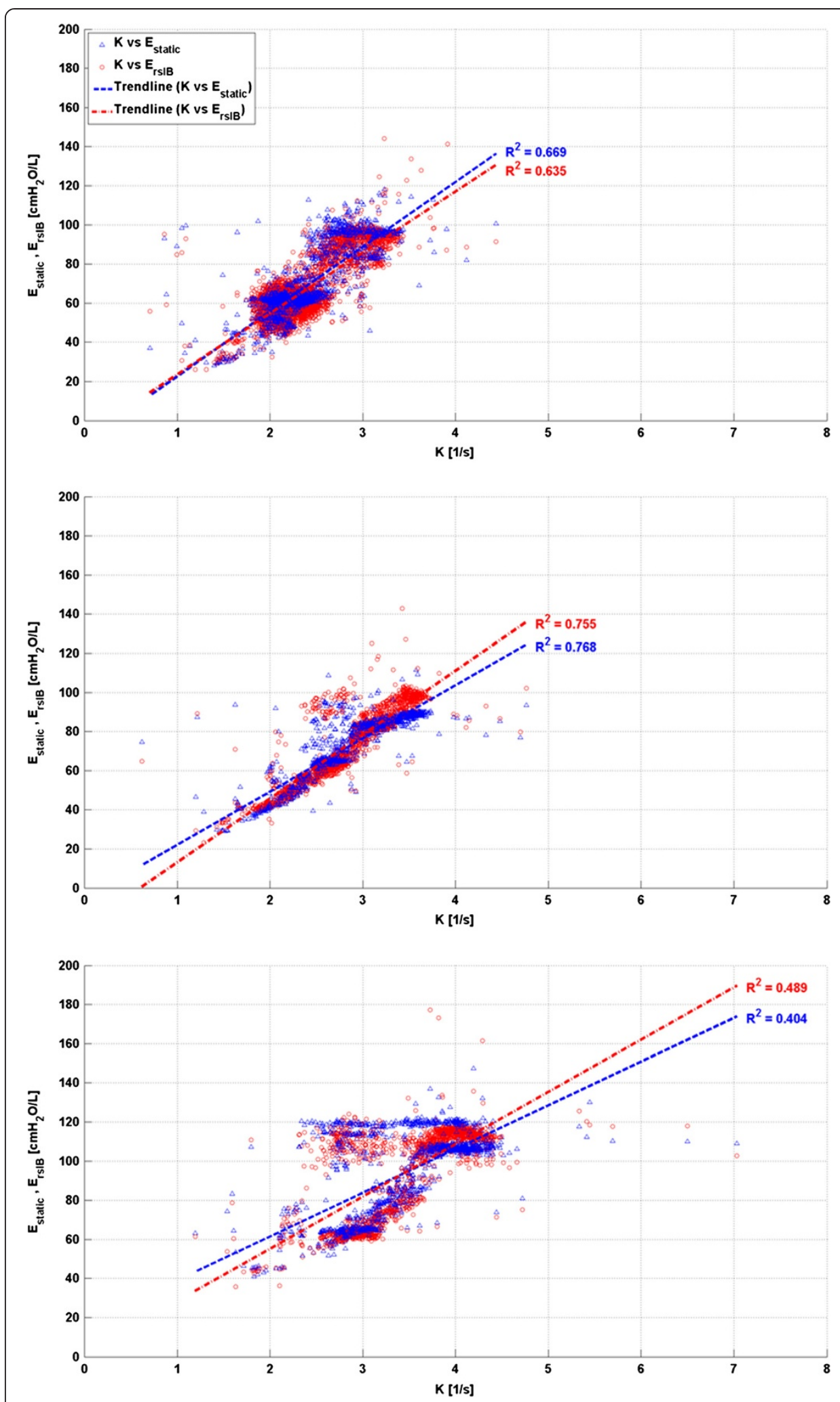

Figure 7 Correlation between $K$ and validation metrics $E_{\text {static }}$ and $E_{r s l B}$. Subject 1 (Top), Subject 2 (Middle) and Subject 3 (Bottom). 
to treatment. Thus, the application of the time-constant model to human patients warrants further investigation.

The EIP method may be erroneous when the automated EIP is too short and does not allow peak pressure to drop to the true plateau pressure [39]. In addition, this simple two-point, static approach may be too simplistic to capture some finer aspects of lung mechanics. Hence, no elastance metric is necessarily a gold standard. This analysis is predominantly based on the comparison of trends where each subject is their own reference. Thus, the best validation of a model is the ability to track clinically expected trends.

The estimation of respiratory resistance and the effect of ARDS on this parameter may be limited. Airway collapse alters respiratory resistance [12,14,19,30]. However, this change is less significant compared to changes in respiratory elastance due to alveolar collapse. A collapsed airway will not have air entering and thus, resistance on expiration will not exist. Equally, a nearly closed airway will have higher resistance. Both hypotheses are potential effects from ARDS, but result in contradiction.

The clinical merit of $K$ relies on the assumption that the respiratory resistance remains constant throughout treatment. Significant variation will result in a poor correlation between $K$ and respiratory elastance. However, the degree to which respiratory resistance varies with PEEP and disease state was different for each of the three subjects in this study. A larger cohort may provide more consistency.

The time-constant model used in this study may not accurately capture regional differences in mechanical properties [16]. Passive expiration could be more accurately modelled using a bi-exponential function, combining the effects of a slower and a faster time-constant to effectively model each lung separately [16,40,41]. However, each time-constant cannot be uniquely distinguished. Therefore, this method could not track disease progression within each lung separately.

\section{Conclusions}

Expiratory data is normally neglected by conventional metrics of respiratory system mechanics. However, the time-constant model provides an alternative means to track changes in disease state throughout treatment to optimise MV. Setting PEEP at minimum elastance, i.e. minimum time-constant, theoretically provides optimal PEEP. In general, the trends obtained using the time-constant model matched those obtained using the EIP method and the integral-based method, demonstrating robustness and potential for guiding MV therapy. However, the assumption of constant resistance leads to less physiological insight.

These are the first results to track and identify clinically relevant and expected pulmonary mechanics breath-to-breath through a clinical recruitment manoeuvre. Such tracking offers insight beyond the metrics and methods presented. Overall, further research is required to confirm the use of such real-time methods in actual ARDS patients, both sedated and spontaneously breathing. However, the ability to identify and track clinically relevant responses to disease progression and MV in real-time shows significant new potential.

Abbreviations

ARDS: Acute respiratory distress syndrome; ICU: Intensive care unit; MV: Mechanical ventilation; PEEP: Positive end expiratory pressure; EIP: End-inspiratory pause; PIP: Peak inspiratory pressure; IQR: Inter-quartile range. 


\section{Authors' contributions}

EJD assisted in the development of the time-constant model, performed the validation and drafted the manuscript. YSC participated in the implementation of the clinical trials, assisted in the development of the time-constant model and helped to draft the manuscript. JGC participated in the implementation and coordination of the study and helped to draft the manuscript. GMS participated in the implementation and coordination of the study. BL and NJ implemented the clinical trials. NSD assisted in the development of the time-constant method. TD participated in the implementation of the clinical trials and the implementation and coordination of the study. All authors read and approved the final manuscript.

\section{Author details}

${ }^{1}$ University of Canterbury, 8041, Christchurch, New Zealand. ${ }^{2}$ Christchurch Hospital, 8011, Christchurch, New Zealand.

${ }^{3}$ University Hospital of Liège, Liège, Belgium. ${ }^{4}$ University of Liège, Liège, Belgium.

Received: 25 March 2013 Accepted: 17 June 2013

Published: 26 June 2013

\section{References}

1. The ADTF: Acute respiratory distress syndrome: The berlin definition. JAMA 2012, 307(23):2526-2533.

2. Gattinoni L, Pesenti A: The concept of "baby lung". Intensive Care Med 2005, 31(6):776-784.

3. Amato MBP, Barbas CSV, Medeiros DM, Magaldi RB, Schettino GP, Lorenzi-Filho G, Kairalla RA, Deheinzelin D, Munoz C, Oliveira R, et al: Effect of a Protective-Ventilation Strategy on Mortality in the Acute Respiratory Distress Syndrome. N Engl J Med 1998, 338(6):347-354.

4. The Acute Respiratory Distress Syndrome Network: Ventilation with Lower Tidal Volumes as Compared with Traditional Tidal Volumes for Acute Lung Injury and the Acute Respiratory Distress Syndrome. N Eng/ J Med 2000, 342(18):1301-1308.

5. McCann UG, Schiller HJ, Carney DE, Gatto LA, Steinberg JM, Nieman GF: Visual validation of the mechanical stabilizing effects of positive end-expiratory pressure at the alveolar level. J Surg Res 2001, 99(2):335-342.

6. Halter JM, Steinberg JM, Schiller HJ, DaSilva M, Gatto LA, Landas S, Nieman GF: Positive End-Expiratory Pressure after a Recruitment Maneuver Prevents Both Alveolar Collapse and Recruitment/Derecruitment. Am J Respir Crit Care Med 2003, 167(12):1620-1626.

7. Lauzon AM, Bates JH: Estimation of time-varying respiratory mechanical parameters by recursive least squares. J Appl Physiol 1991, 71(3):1159-1165.

8. Sundaresan A, Yuta T, Hann CE, Geoffrey Chase J, Shaw GM: A minimal model of lung mechanics and model-based markers for optimizing ventilator treatment in ARDS patients. Comput Meth Prog Biomed 2009, 95(2):166-180.

9. Ma B, Bates J: Modeling the Complex Dynamics of Derecruitment in the Lung. Ann Biomed Eng 2010, 38(11):3466-3477

10. Sundaresan A, Chase JG: Positive end expiratory pressure in patients with acute respiratory distress syndrome - The past, present and future. Biomed Signal Proce 2011, 7(2):93-103.

11. Al-Rawas N, Banner M, Euliano N, Tams C, Brown J, Martin AD, Gabrielli A: Expiratory time constant for determinations of plateau pressure, respiratory system compliance, and total resistance. Crit Care 2013, 17(1):R23.

12. Carvalho A, Jandre F, Pino A, Bozza F, Salluh J, Rodrigues R, Ascoli F, Giannella-Neto A: Positive end-expiratory pressure at minimal respiratory elastance represents the best compromise between mechanical stress and lung aeration in oleic acid induced lung injury. Crit Care 2007, 11(4):R86.

13. Lucangelo U, Bernabè F, Blanch L: Lung mechanics at the bedside: make it simple. Curr Opin Crit Care 2007, 13(1):64-72

14. Suarez-Sipmann F, Bohm SH, Tusman G, Pesch T, Thamm O, Reissmann H, Reske A, Magnusson A, Hedenstierna G: Use of dynamic compliance for open lung positive end-expiratory pressure titration in an experimental study. Crit Care Med 2007, 35:214-221.

15. Lambermont B, Ghuysen A, Janssen N, Morimont P, Hartstein G, Gerard P, D'Orio V: Comparison of functional residual capacity and static compliance of the respiratory system during a positive end-expiratory pressure (PEEP) ramp procedure in an experimental model of acute respiratory distress syndrome. Crit Care 2008, 12(4):R91

16. Bates JHT: Lung Mechanics: An Inverse Modeling Approach. New York: Cambridge University Press; 2009.

17. Moller K, Zhao Z, Stahl C, Guttmann J: Seperate analysis of respiratory system mechanics in inspiration and expiration. In 55 GMDS-Jahrestagung: 2010; Mannheim. Germany: Antares Verlag; 2010:566-567.

18. Moller K, Zhao Z, Stahl CA, Guttmann J: On the analysis of dynamic lung mechanics separately in ins- and expiration. In MEDICON 2010, IFMBE Proceedings: 2010. Chalkidiki, Greece: Springer; 2010:164-167.

19. Chiew YS, Chase JG, Shaw G, Sundaresan A, Desaive T: Model-based PEEP Optimisation in Mechanical Ventilation. Biomed Eng Online 2011, 10(1):111.

20. Chiew YS, Chase JG, Lambermont B, Janssen N, Schranz C, Moeller K, Shaw GM, Desaive T: Physiological relevance and performance of a minimal lung model - an experimental study in healthy and acute respiratory distress syndrome model piglets. BMC Pulm Med 2012, 12:59-59.

21. Ingelstedt S, Jonson B, Nordström L, Olsson S-G: A Servo-Controlled Ventilator Measuring Expired Minute Volume, Airway Flow and Pressure. Acta Anaesthesiol Scand 1972, 16:7-27.

22. Fuleihan SF, Wilson RS, Pontoppidan H: Effect of Mechanical Ventilation with End-inspiratory Pause on Blood-Gas Exchange. Anesth Analg 1976, 55(1):122-130.

23. Pillet $\mathrm{O}$, Choukroun ML, Castaing Y: Effects of inspiratory flow rate alterations on gas exchange during mechanical ventilation in normal lungs, Efficiency of end-inspiratory pause. CHEST Journal 1993, 103(4):1161-1165.

24. Hann CE, Chase JG, Lin J, Lotz T, Doran CV, Shaw GM: Integral-based parameter identification for long-term dynamic verification of a glucose-insulin system model. Comput Methods Programs Biomed 2005, 77(3):259-270.

25. Ballard-Croft C, Wang D, Sumpter LR, Zhou X, Zwischenberger JB: Large-Animal Models of Acute Respiratory Distress Syndrome. Ann Thorac Surg 2012, 93(4):1331-1339. 
26. Ware LB: Modeling human lung disease in animals. Am J Physiol Lung Cell Mol Physiol 2008, 294(2):L149-L150.

27. Bastarache JA, Blackwell TS: Development of animal models for the acute respiratory distress syndrome. Dis Model Mech 2009, 2(5-6):218-223.

28. Schuster D: ARDS: clinical lessons from the oleic acid model of acute lung injury. Am J Respir Crit Care Med 1994, 149(1):245-260.

29. Chiew YS, Desaive T, Lambermont B, Janssen N, Shaw GM, Schranz C, Mishra A, Damanhuri N, Chase J: Performance of lung recruitment model in healthy anesthetized pigs, 2012 World Congress of Medical Physics and Biomedical Engineering. Beijing, China; 2012:1. May 26-31, 2012.

30. Mols G, Kessler V, Benzing A, Lichtwarck-Aschoff M, Geiger K, Guttmann J: Is pulmonary resistance constant, within the range of tidal volume ventilation, in patients with ARDS? Br J Anaesth 2001, 86(2):176-182.

31. Albert SP, DiRocco J, Allen GB, Bates JHT, Lafollette R, Kubiak BD, Fischer J, Maroney S, Nieman GF: The role of time and pressure on alveolar recruitment. J Appl Physiol 2009, 106(3):757-765.

32. Barbas CSV, De Matos GFJ, Pincelli MP, Da Rosa Borges E, Antunes T, De Barros JM, Okamoto V, Borges JB, Amato MBP, De Carvalho CR R: Mechanical ventilation in acute respiratory failure: recruitment and high positive end-expiratory pressure are necessary. Curr Opin Crit Care 2005, 11(1):18-28.

33. Ganzert S, Moller K, Steinmann D, Schumann S, Guttmann J: Pressure-dependent stress relaxation in acute respiratory distress syndrome and healthy lungs: an investigation based on a viscoelastic model. Crit Care 2009, 13(6):R199.

34. Andreassen S, Steimle KL, Mogensen ML, Serna JB, Rees S, Karbing DS: The effect of tissue elastic properties and surfactant on alveolar stability. J Appl Physiol 2010, 109(5):1369-1377.

35. Zhao Z, Steinmann D, Frerichs I, Guttmann J, Moller K: PEEP titration guided by ventilation homogeneity: a feasibility study using electrical impedance tomography. Crit Care 2010, 14:R8.

36. Grinnan D, Truwit J: Clinical review: Respiratory mechanics in spontaneous and assisted ventilation. Crit Care 2005, 9(5):472-484

37. Khirani S, Polese G, Aliverti A, Appendini L, Nucci G, Pedotti A, Colledan M, Lucianetti A, Baconnier P, Rossi A: On-line monitoring of lung mechanics during spontaneous breathing: a physiological study. Respir Med 2010, 104(3):463-471.

38. Talmor D, Sarge T, Malhotra A, O'Donnell CR, Ritz R, Lisbon A, Novack V, Loring SH: Mechanical Ventilation Guided by Esophageal Pressure in Acute Lung Injury. N Engl J Med 2008, 359(20):2095-2104.

39. Barberis L, Manno E, Guérin C: Effect of end-inspiratory pause duration on plateau pressure in mechanically ventilated patients. Intensive Care Med 2003, 29(1):130-134.

40. Chelucci GL, Dall' Ava-Santucci J, Dhainaut JF, Chelucci A, Allegra A, Paccaly D, Brunet F, Milic-Emili J, Lockhart A: Modelling of passive expiration in patients with adult respiratory distress syndrome. Eur Respir J 1993, 6(6):785-790

41. Chelucci G-L, Locchi F, Zin WA: On the interaction between respiratory compartments during passive expiration in ARDS patients. Respir Physiol Neurobiol 2005, 145(1):53-63.

doi:10.1186/1475-925X-12-57

Cite this article as: van Drunen et al:: Expiratory model-based method to monitor ARDS disease state. BioMedical Engineering OnLine 2013 12:57.

\section{Submit your next manuscript to BioMed Central and take full advantage of:}

- Convenient online submission

- Thorough peer review

- No space constraints or color figure charges

- Immediate publication on acceptance

- Inclusion in PubMed, CAS, Scopus and Google Scholar

- Research which is freely available for redistribution

Submit your manuscript at www.biomedcentral.com/submit 